\section{A MONOZYGOTIC COTWIN CONTROL STUDY \\ OF A TREATMENT \\ FOR MYOPIA}

\section{J. THEODORE SCHWARTZ}

National Eye Institute, NIH, Bethesda, Maryland, USA

Myopia is by far the world's most common cause of defective vision. Methods of control have been directed toward limiting accommodation and the effort of near work. This is a description of a prospective cotwin control study undertaken to assess the effect of a specific treatment in retarding the progression of myopia.

Twenty-five pairs of twins are participating in this three-year study. Basic requirements for participation include monozygosity, as determined by blood serotyping and anthropometric similarity, age 6 to 14 at time of entry into the study, and myopic refraction for each member of the twinship. All participating twins were selected from members of the Twin Register for Eye Examinations in the metropolitan Washington, D.C. area, which was described at the First International Symposium on Twin Studies. One member of each twinship wears a standard myopic spectacle correction as determined by refraction under cycloplegia. The cotwin wears specially prescribed bifocal lenses and instills cycloplegic eye drops in each eye once daily. Random assignment of standard spectacle correction or special treatment status for members of each twinship was established by protocol.

All twins are reexamined at 6-month intervals, at which time spectacle corrections are updated as indicated. Base line and final examinations include keratometry and the measurement of intraocular compartments by ultrasonic techniques in addition to refraction.

The data collection phase is in its final year.

J. T. Schwartz, M.D., National Eye Institute, NIH, Bethesda, Maryland 20014, USA

\section{TRACHEOBRONCHIAL CLEARANCE \\ IN MZ AND DZ TWINS EXPOSED TO ENVIRONMENTAL AGENTS}

\section{PER CAMNER}

\author{
Department of Environmental Hygiene, The Karolinska \\ Institute, Stockholm, Sweden
}

The tracheobronchial mucosa is provided with ciliated epithelium. The cilia drive a mucus blanket up to the pharynx whereafter the mucus is swallowed. This defense mechanism has been studied by letting subjects inhale a test aerosol of $6 \mu \mathrm{m}$ teflon particles (specific density $2 \mathrm{~g} / \mathrm{cc}$ ) tagged with a radionuclide and external measurement of the radioactivity in the lungs for two hours. Well reproducible clearance patterns were obtained within one and the same subject, but the patterns differed very much from one subject to another.

In 9 pairs of $\mathrm{MZ}$ twins the clearance patterns were highly similar within the pairs, but not within 9 pairs of DZ twins. This indicates that the large differences in the clearance patterns are to an important extent genetically determined.

As the clearance patterns were highly similar within pairs of $M Z$ twins, a long-term effect of an agent on clearance should preferably be studied in $\mathrm{MZ}$ pairs discordant with regard to the agent. In Io pairs of smoking-discordant MZ pairs clearance was on the average slower in the smokers. In another study $\mathrm{I}_{5} \mathrm{MZ}$ pairs were examined, one in each pair having lived for the last Io years in Stockholm and the partner having resided during the same time in a rural area or a small town. On the average, no differences within the pairs could be seen. There are also large differences in the mucociliary transport velocity in the nose. An almost significant correlation of the mucus flow rate (studied by a saccharine particle) between the two members in 12 pairs of $\mathrm{MZ}$ twins was found. This suggests that genetic factors are at least partly responsible for the large difference in mucociliary transport in the nose.

Prof. Per Camner, Department of Environmental Hygiene, The Karolinska Institute, S-104 or Stockholm, Sweden 